\title{
The Impact of Nuclear Star Formation on Gas Inflow to AGN
}

\author{
R. I. Davies ${ }^{1}$, E. Hicks ${ }^{1}$, M. Schartmann ${ }^{1,2}$, R. Genzel ${ }^{1}$, \\ L. J. Tacconi ${ }^{1}$, H. Engel ${ }^{1}$, A. Burkert ${ }^{1,2}$, M. Krause ${ }^{1,2}$, A. Sternberg ${ }^{3}$, \\ F. Mueller Sánchez ${ }^{4}$, and W. Maciejewski ${ }^{5}$ \\ ${ }^{1}$ Max-Planck-Institut für extraterrestrische Physik, Garching, Germany \\ Email: davies@mpe.mpg.de \\ ${ }^{2}$ Universitäts-Sternwarte München, Germany \\ ${ }^{3}$ School of Physics and Astronomy, Tel Aviv University, Tel Aviv, Israel \\ ${ }^{4}$ Instituto de Astrofisica de Canarias, La Laguna, Tenerife, Spain \\ ${ }^{5}$ Astrophysics Research Institute, Liverpool John Moores University, UK
}

\begin{abstract}
Our adaptive optics observations of nearby AGN at spatial resolutions as small as $0 .{ }^{\prime \prime} 085$ show strong evidence for recent, but no longer active, nuclear star formation. We begin by describing observations that highlight two contrasting methods by which gas can flow into the central tens of parsecs. Gas accumulation in this region will inevitably lead to a starburst, and we discuss the evidence for such events. We then turn to the impact of stellar evolution on the further inflow of gas by combining a phenomenological approach with analytical modelling and hydrodynamic simulations. These complementary perspectives paint a picture in which all the processes are ultimately regulated by the mass accretion rate into the central hundred parsecs, and the ensuing starburst that occurs there. The resulting supernovae delay accretion by generating a starburst wind, which leaves behind a clumpy interstellar medium. This provides an ideal environment for slower stellar outflows to accrete inwards and form a dense turbulent disk on scales of a few parsecs. Such a scenario may resolve the discrepancy between the larger scale structure seen with adaptive optics and the small-scale structure seen with VLTI.
\end{abstract}

Keywords. galaxies: active, galaxies: kinematics and dynamics, galaxies: nuclei, galaxies: Seyfert, galaxies: starburst, infrared: galaxies

\section{Introduction}

Many studies of AGN, particularly those concerned with understanding the co-evolution of black holes and their host galaxies through cosmic time, rely on observations of quasars. The reason is simply that these are the most luminous of such objects and can be studied at all redshifts. While they form an important component of the AGN population, the luminosity function (Hao et al. 2005) indicates that the number density of lower-luminosity AGN is orders of magnitude greater. This is particularly true in the local universe because of the effects of downsizing, which means that at low redshift black hole growth occurs primarily in AGN with black hole masses $<10^{8} M_{\odot}$ (Best et al. 2005) and Seyfert-like luminosities (Hasinger et al. 2005). As a result, with only a few exceptions such as Mkn 231 (Davies et al. 2004), detailed studies of nearby AGN have to focus on the lower-luminosity AGN such as Seyferts.

This has important implications. Although most quasar activity may be fuelled via mergers of gas rich galaxies, Seyfert nuclei typically reside in (preferentially early type) spiral galaxies (Ho 2008). As a result, Seyfert activity is likely to be fuelled via secular processes associated with disk evolution such as those outlined by Shlosman et al. (1990) 
and Wada (2004). One obvious part of the overall scheme is the role played by bars, which are known to drive gas inwards from the large-scale disk. Yet despite the many attempts to find a correlation between the presence of a bar and an AGN, the statistical outcome is, at best, for only a marginal link between these phenomena. This, perhaps, should not be surprising. Bars drive large amounts of gas inwards (of order $0.1 M_{\odot} \mathrm{yr}^{-1}$; Regan \& Teuben 2004), working on spatial scales of kpcs and timescales of Gyrs. In contrast, a Seyfert nucleus requires typically $\lesssim 0.01 M_{\odot} \mathrm{yr}^{-1}$ of gas (Jogee et al. 2006), and the relevant temporal and spatial scales are Myr and pc. The two regimes are orders of magnitude different in every respect. Thus, while it is well understood how bars can create a gas reservoir and a starburst in the central kiloparsec (and there is plenty of observational evidence to support the link between bars and both of these phenomena: Sakomoto et al. 1999, Laurikainen et al. 2004, and Jogee et al. 2005 are a few examples among many), this is only a single possible step in the process that leads to AGN fuelling.

It is now understood that the properties of the central $\mathrm{kpc}$ - the circumnuclear region - can play an important role in driving gas further inwards. But although observations have yielded many tantalising results, reaching definitive conclusions is difficult. For example, Martini et al. (2003) looked at the detailed circumnuclear dust morphologies of matched samples of active and inactive galaxies. One of their results was that AGN are only found in galaxies that exhibit dusty spiral patterns. But they also found that spiral patterns of any sort were associated equally often with active and inactive galaxies. This, again, is likely a result of the differing spatial and temporal scales of the phenomena. The aspect of time variability is one of the issues addressed by NUGA (García-Burillo et al. 2007), a study of the distribution and kinematics of the molecular gas in nearby low luminosity AGN. One of their key results was that gravitational torques can not only drive gas in to form a circumnuclear ring, but also tend to drive gas out from the nuclear region towards the ring. This led García-Burillo et al. (2005) to propose a scenario in which further inward flow was possible episodically if two conditions were fulfilled: the bar should be weakened by dynamical feedback, and the viscosity should increase due to a sufficiently steep density gradient in the circumnuclear ring.

These examples show that there is still much we do not understand about the conditions under which gas can be transported inward from kpc scales to the nuclear region, and it is one of the topics addressed here: we describe two highly contrasting case studies where gas is being driven inwards to the central tens of parsecs. We also show evidence that, once there, the gas is likely to trigger a starburst. We also discuss the impact of the starburst on the fate of the gas, and a possible link to the sub-parsec scales.

\section{Watching Gas Flow Inwards}

In this section, we describe contrasting cases where gas can be observed as it flows in towards an AGN. Although we present only two examples here, analyses of noncircular motions and gas inflow mechanisms on similar scales have been performed for other galaxies as well: NGC 7469 (Davies et al. 2004), NGC 6951 (Storchi-Bergmann et al. 2007), NGC 4051 (Riffel et al. 2008), NGC 7582 (Riffel et al. 2009), and NGC 4151 (Storchi-Bergmann et al. 2009).

In the case of NGC 1068 (Mueller Sánchez et al. 2009), the inflow rate is prodigious, due to chance events, and probably cannot be sustained. In the other, NGC 1097 (Davies et al. 2009), the inflow is much more moderate and appears to be regulated in a way that is sustainable for timescales of a Gyr. Intriguingly, in neither case do we see gas flowing all the way down to the AGN: the inflow is terminated at spatial scales of a few to tens of parsecs, where it is instead expected to fuel a starburst. 

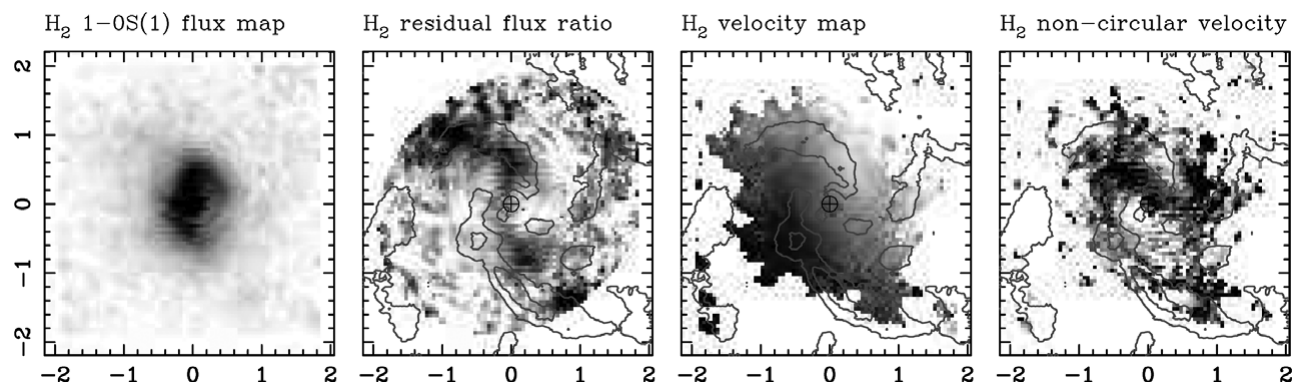

Figure 1. Flux and velocity maps of the 1-0 S(1) $\mathrm{H}_{2}$ emission line in NGC 1097. Far and center left: flux map and residual after subtracting elliptical isophotes, showing the three photometric arms. Center and far right: velocity field and residual after subtracting circular motions, showing the two kinematic arms. Adapted from Davies et al. (2009).

\subsection{Gas Inflow in NGC 1068}

Among the nearby AGN we have studied (Davies et al. 2007; Hicks et al. 2009), NGC 1068 is rather unusual. The $\mathrm{H}_{2}$ emission in the central $250 \mathrm{pc}$ originates in an expanding offcentre ring or shell with particularly bright and massive clumps around the north east side. Filaments of gas extend from the ring at a radius of about $30 \mathrm{pc}$ to the AGN on both sides. Mueller Sánchez et al. (2009) has modelled the morphology and kinematics of the filaments, finding that the only way to simultaneously account for both constraints is if the filaments trace gas that is falling almost directly in towards the AGN. These models indicate that the infall timescale is about 1.3 Myr. One of the filaments lies across the front of the AGN, suggesting that inelastic collisions may allow the gas to settle on scales of a few parsecs.

Estimating the mass of gas in the filaments is extremely difficult. Mueller Sánchez et al. (2009) used a variety of methods to derive a mass of $\sim 2 \times 10^{7} M_{\odot}$, although with about a factor of 3 uncertainty. Thus the inflow rate to the central few parsecs is of order $15 M_{\odot} \mathrm{yr}^{-1}$. This is a remarkably high rate, 2-3 orders of magnitude greater than that needed to power the AGN itself. And because the characteristics of the gas in the central region are so unusual, and the inflow appears to be due to chance combination of circumstances, such a rapid inflow is probably unsustainable.

\subsection{Gas Inflow in NGC 1097}

NGC 1097 exhibits many classical features of disk galaxies: a large-scale bar, a circumnuclear ring and starburst, an inner spiral, and a low-luminosity AGN. Its one peculiarity is that the inner spiral has three photometric arms (Prieto et al. 2005). Integral field observations (Davies et al. 2009) showed that this pattern is due to obscuration of stellar light by gas and dust in the molecular arms. Intriguingly, as seen in Figure 1, the gas kinematics reveal a strong non-circular velocity residual in the form of a two-arm spiral - as expected from linear theory which indicates that the projected line-of-sight velocity pattern of an $m$-arm spiral is an $(m-1)$-arm spiral. The properties of the arms allow one to derive an inflow rate along them of $\sim 1.2 M_{\odot} \mathrm{yr}^{-1}$. However, hydrodynamical simulations of nuclear spirals show that there is also significant outflow between the arms in the low-density regions (gas from the arms overshoots the nucleus and continues as a diverging outflow). In the simulations, the net inflow rate is a factor 20 less; applying this to NGC 1097 means it could be as little as $0.06 M_{\odot} \mathrm{yr}^{-1}$.

Given that there is of order $10^{8} M_{\odot}$ of gas within a radius of $700 \mathrm{pc}$, such a modest inflow rate would require $1.8 \mathrm{Gyr}$ to drain the gas reservoir in the circumnuclear region. If this gas is replenished by the ring, which itself is fed by the large-scale bar, then the 

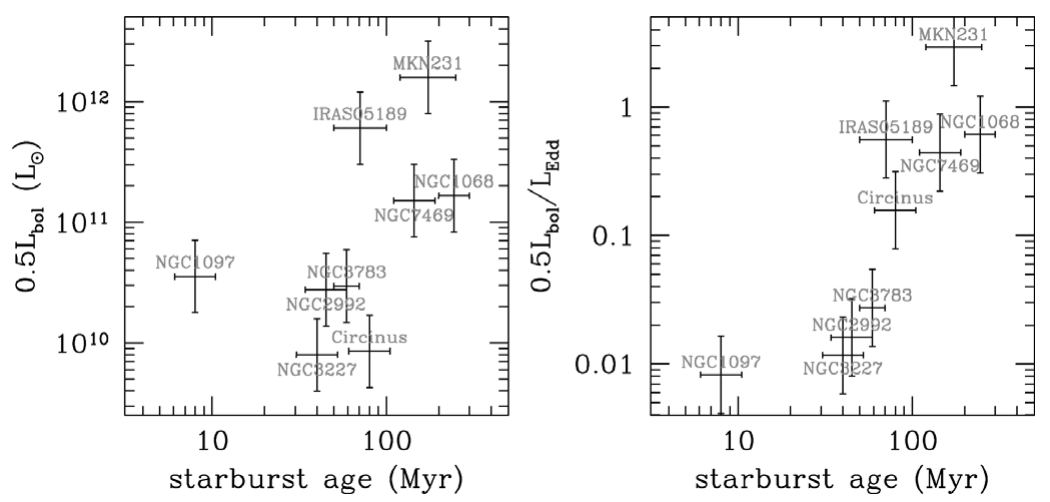

Figure 2. Relation between the characteristic age of most recent starburst and the AGN luminosity and accrection rate. This figure is adapted from Davies et al. (2007) and includes an additional point for NGC 2992 from Friedrich et al. (2009).

inflow could in principle continue even longer. Thus the inner spiral in NGC 1097 appears to be driving gas inwards at a sustainable rate.

As for NGC 1068, the gas is not reaching the AGN. Instead the spiral arms appear to terminate in the central 10-20 pc, at which point the gas dispersion increases dramatically (Davies et al. 2007; Hicks et al. 2009). If the gas piles up here, it will inevitably lead to a starburst - and a nuclear starburst has been reported (Storchi-Bergmann et al. 2005; Davies et al. 2007). The estimated gas inflow rate could lead to episodic starbursts similar to this one every $20-150 \mathrm{Myr}$.

\section{Nuclear Starbursts}

We have seen in the previous section how, at least for these two cases, gas flows into the central few to tens of parsecs. If it is stalled here, one would naturally expect it to trigger a starburst. Evidence for starbursts on these scales was presented by Davies et al. (2007), who used near infrared integral field spectroscopy combined with adaptive optics to achieve high spatial resolution. Using the spectral information, these authors were able to separate out the non-stellar continuum (hot dust emission) associated with the AGN, and show that in every case the stellar continuum itself was spatially resolved with a typical size scale $<50$ pc. Furthermore, they showed that this was a distinct stellar population, younger and dynamically cooler than the bulge: photometrically, from an excess in the stellar continuum above an $r^{1 / 4}$ law fitted to $1-2^{\prime \prime}$ scales and extrapolated inwards; and kinematically, from a drop in the stellar dispersion on the same spatial scales. Constraints on stellar population synthesis models provided by several independent diagnostics indicated that the starburst ages were young, lying in the range 10-300 Myr. One important piece of evidence was the remarkably low equivalent width of the $\operatorname{Br} \gamma$ line, which shows unambiguously that the star formation has ceased: i.e. although still young, these are post-starbursts. Intriguingly, starburst models imply that during the short (perhaps only $10 \mathrm{Myr}$ ) actively star forming phase, the luminosity of the nuclear starburst would have been an order of magnitude greater than it is now - with a star formation rate per unit area comparable to that in ULIRGs. This high intensity appears to make sense in terms of the high gas-mass surface density which, from the Kennicutt-Schmidt law, is expected to lead to high star-formation rates.

More pertinent to the issue of gas inflow, the authors showed that there appears to be a relation between the characteristic age of the most recent episode of star formation 
and the luminosity of, or accretion rate onto, the AGN (Figure 2). This figure suggests that there is a delay of 50-100 Myr between the short-duration starburst and the onset of accretion onto the AGN. This timescale is interesting because it corresponds to the age at which, for a short burst, one expects the type II supernovae phase to be finishing. This topic is discussed in more detail in Section 4, which addresses the impact of stellar winds and supernovae on gas inflow and outflow, and how these might provide a link between phenomena observed with adaptive optics on $\sim 10 \mathrm{pc}$ scales and those seen with infrared interferometric techniques on $0.1-1$ pc scales.

\section{Stellar Outflows and the Torus: Linking $10 \mathrm{pc}$ and $1 \mathrm{pc}$ Scales}

The data presented by Davies et al. (2007) and Hicks et al. (2009) show that nuclear star formation is occuring on the same spatial scales, up to a few tens of parsecs, as the gas distribution, and that on these scales the two components have similar kinematics. This means the stars and gas must be mixed. It is inevitable that the star formation will have some impact on the gas, and this was argued phenomenologically by Davies et al. (2007). Hydrodynamical simulations of the impact of stellar evolution, such as those presented in Schartmann et al. (2009) give a clearer picture. To understand this in the context of Seyfert nuclei, these simulations have been scaled to match a typical starburst there, as described by Schartmann et al. (these proceedings): $M_{\mathrm{BH}} \sim 10^{7} M_{\odot}$, $M_{\text {stars }} \sim 2 \times 10^{8} M_{\odot}$ out to $50 \mathrm{pc}$, a dispersion $\sigma_{*} \sim 100 \mathrm{~km} \mathrm{~s}^{-1}$ plus rotation, and a massloss rate $\sim 0.1 M_{\odot} \mathrm{yr}^{-1}$ corresponding to an age of $50 \mathrm{Myr}$ but which of course would vary with time. The simulations show that there are two fairly well-defined regimes separated by a stellar outflow speed of about $250 \mathrm{~km} \mathrm{~s}^{-1}$. Fast outflows (which could represent winds from OB stars, as well as type II and type I supernovae) lead to a starburst wind which efficiently removes the diffuse ISM. Slower outflows (which represent, for example, planetary nebulae or winds from AGB stars) lead to a large number of clumps that can accrete efficiently to smaller scales, giving rise to a dense and turbulent disk with a radius of $0.5-1 \mathrm{pc}$. Taken together, these imply that there is a phase in a starburst when accretion to smaller scales is possible and efficient. For a short starburst, this phase would begin after about $50 \mathrm{Myr}$, once there are no more OB stars or type II supernovae; and it would end after about $300 \mathrm{Myr}$ once type I supernovae start to appear.

The size scale of the central disk is similar to that of the compact $8-12 \mu \mathrm{m}$ structure seen in NGC 1086 (the galaxy to which the simulation is scaled) by Raban et al. (2009) using MIDI on the VLTI; and within a factor of a few of the compact structure seen in Circinus by Tristram et al. (2007) using the same instrument. As already suggested by Davies et al. (2008), these simulations imply that stellar outflows are a link between the gas structure seen on scales of $10-50 \mathrm{pc}$ using adaptive optics, and those seen on $0.1-1 \mathrm{pc}$ scales using infrared interferometry. This scenario has a number of similarities to the simulations of Wada et al. (2009). Although the initial conditions and the distribution of energy input are rather different, the result is a vertically thick (up to $10 \mathrm{pc}$ from the disk plane) distribution at large radii, and in the inner few parsec a compact turbulent disk.

If one considers either of these structures to be associated with the molecular obscuring torus, then one must also conclude that they both are. As illustrated in Figure 3 and stated by Hicks et al. (2009), this implies that the torus comprises multiple components each of which fulfills different roles; that it must be a star-forming torus; and, because the starbursts are episodic, that it must be a dynamical entity. What we see on 10$50 \mathrm{pc}$ scales is the global structure, a diffuse and clumpy envelope where there are short repeated bursts of star formation. The slow stellar winds accrete down to smaller $\sim 1 \mathrm{pc}$ 


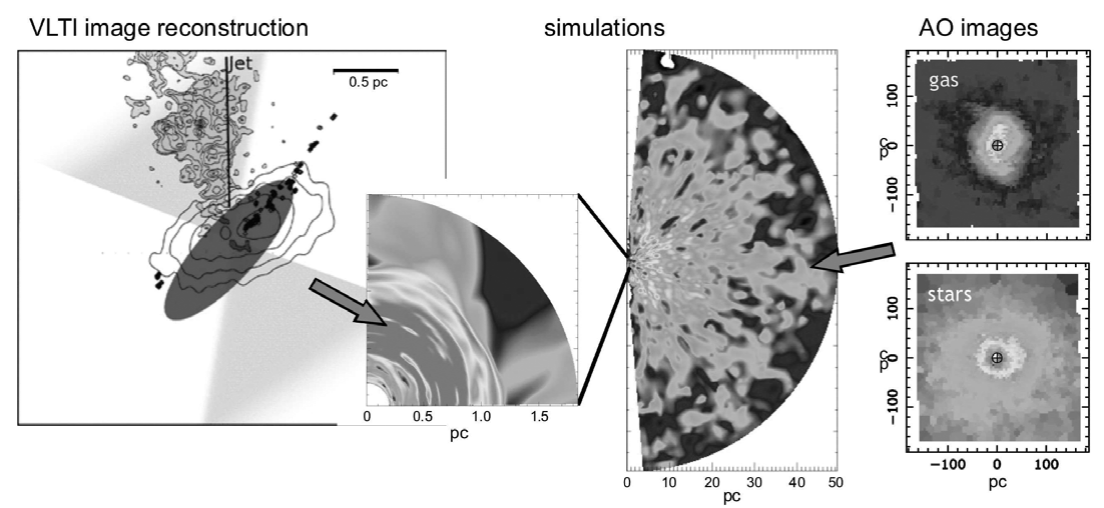

Figure 3. Composite figure showing how hydrodynamical simulations of stellar outflows might provide a link between the $10 \mathrm{pc}$ and $1 \mathrm{pc}$ scale structure within the torus. Panels are taken from Raban et al. (2009), Hicks et al. (2009), Davies et al. (2009), and simulations similar to those in Schartmann et al. (2009) but scaled to a typical Seyfert nucleus. This figure is illustrative, and the individual panels do not necessarily refer to the same specific object.

scales where they form the more compact structure, a dense and turbulent disk. The evolution of this disk, in terms of further star formation and gas inflow, is addressed by Schartmann et al. (these proceedings).

One final point worth noting is the similarity between these simulations and those performed for the Galactic Center, on scales about 100 times smaller, by Cuadra et al. (2006) and Cuadra et al. (2008). Both simulations were performed within a 0.5 pc volume in which the stars, their orbits, and their outflows, matched as precisely as possible the known configuration at that time. In the earlier version, where there were both slow $\sim 200 \mathrm{~km} \mathrm{~s}^{-1}$ and fast $\sim 700 \mathrm{~km} \mathrm{~s}^{-1}$ stellar winds, the result was that much of the ISM was blown out but that clumps of cooler dense gas accreted to form a compact disk that extended out to $\sim 0.04 \mathrm{pc}$. In the later version, the updated stellar wind speeds were all fast, and nearly the entire ISM was blown out. It is reassuring that qualitatively, these simulations yield the same result as those of Schartmann et al. (2009) even though the scales are drastically different, because in both cases it is the same processes (i.e., stellar outflows) that govern the evolution of the region.

\section{Conclusions}

We have presented a brief review of the current status of our work on the impact that star formation has on gas inflow to Seyfert nuclei. We are not yet able to follow gas all the way down to the AGN itself, and instead we have focussed on spatial scales from a few hundred parsecs down to the central parsec. Our main conclusions are:

- We have seen two contrasting methods of gas inflow that feed a nuclear starburst. While showing how gas might reach the central few to tens of parsecs, this also raises questions about the sustainability of inflows, how far in they reach, and whether it is possible to define what a typical inflow mechanism or rate is.

- There are short intense starbursts in the central tens of parsecs around Seyfert nuclei. In general, starbursts will be an inevitable consequence of gas inflow.

- Stellar outflows play a key role in the evolution of the ISM on these scales. Specifically, fast outflows tend to blow out the diffuse ISM, while slow outflows can lead to efficient accretion of gas onto smaller scales. This may be a link between what is seen 
on $10-50$ pc scales with adaptive optics, and what is observed on $0.1-1$ pc scales with infrared interferometry.

- The molecular obscuring torus most likely consists of multiple structures on different scales that fulfil different roles. Since the state of the torus is strongly affected by the episodic star formation that occurs within it, we need to think of it as a dynamic entity.

\section{References}

Best, P., Kauffmann, G., Heckman, T., Birnchmann, J., Charlot, S., Iverzić, Ž., \& White, S. 2005, MNRAS, 362, 25

Cuadra, J., Nayakshin, S., Springel, V., \& Di Matteo, T. 2006, MNRAS, 366, 358

Cuadra, J., Nayakshin, S., \& Martins, F. 2008, MNRAS, 383, 458

Davies, R., Tacconi, L., \& Genzel, R. 2004a, ApJ, 602, 148

Davies, R., Tacconi, L., \& Genzel, R. 2004b, ApJ, 613, 781

Davies, R., Mueller Sanchez, D., Genzel, R., Tacconi, L., Hicks, E., Friedrich, S., \& Sternberg A. $2007, A p J, 671,1388$

Davies, R. 2008, New Astron. Revs., 52, 307

Davies, R., Maciejewski, W., Hicks, E., Tacconi, L., Genzel, R., \& Engel, H. 2009, ApJ, 702, 114

Friedrich, S., Davies, R., Hicks, E., Engel, H., Mueller Sánchez, F., Genzel, R., \& Tacconi, L. 2009, $A \mathscr{E} A$, submitted

García-Burillo, S., Combes, F., Schinnerer, E., Boone, F., \& Hunt, K. 2005 A\& A, 441, 1011

García-Burillo, S., Combes, F., Usero, A., \& Gracía-Carpio, J. 2007, New Astron. Revs., 51, 160

Hasinger, G., Miyaji, T., \& Schmidt, M. 2005, A\&A, 441, 417

Hao, L., et al. 2005, AJ, 129, 1795

Hicks, E., Davies, R., Malkan, M., Genzel, R., Tacconi, L., Mueller Sánchez, F., \& Sternberg, A. $2009, A p J, 696,448$

Ho, L. 2008, ARAA, 46, 475

Jogee, S., Scoville, N., \& Kenney, J. 2005, ApJ, 630, 837

Jogee, S. 2006, Lecture Notes in Physics, 693, 143

Laurikainen, E., Salo, H., \& Buta, R. 2004, ApJ, 607, 103

Martini, P., Regan, M., Mulchaey, J., \& Pogge, R. 2003, ApJ, 589, 774

Mueller Sánchez, F., Davies, R., Genzel, R., Tacconi, L., Eisenhauer, F., Hicks, E., Friedrich, S., \& Sternberg, A. 2009, ApJ, 691, 749

Prieto, A., Maciejewski, W., \& Reunanen, J. 2005, AJ, 130, 1472

Raban, D., Jaffe, W., Röttgering, H., Meisenheimer, K., \& Tristram, K. 2009, MNRAS, 394, 1325

Regan, M. \& Teuben, P. 2004, ApJ, 600, 595

Riffel, R., Storchi-Bergmann, T., Winge, C., McGregor, P., Beck, T., \& Schmitt, H. 2008, MNRAS, 385, 1129

Riffel, R., Storchi-Bergmann, T., Dors, O., \& Winge, C. 2009, MNRAS, 393, 783

Sakamoto, K., Okumura, S., Ishizuki, S., \& Scoville, N. 1999, ApJ, 525, 691

Schartmann, M., Meisenheimer, K., Klahr, H., Camenzind, M., Wolf, S., \& Henning, T. 2009, MNRAS, 393, 759

Shlosman, I., Begelman, M., \& Frank, J. 1990, Nature, 345, 679

Storchi-Bergmann, T., Nemmen, R., Spinelli, P., Eracleous, M., Wilson, A., Filippenko, A., \& Livio, M. 2005, ApJ, 624, L13

Storchi-Bergmann, T., Dors, O., Riffel, R., Fathi, K., Axon, D., Robinson, A., Marconi, A., \& Östlin, G. 2007, ApJ, 670, 959

Storchi-Bergmann, T., Simões-Lopes, R., McGregor, P., Riffel, R., Beck, T., \& Martini, P. 2009, $M N R A S$, in press

Tristram, K., et al. 2007, A\&BA, 474, 837

Wada, K. 2004, in Coevolution of Black Holes and Galaxies, ed. L. C. Ho (Cambridge: Cambridge University Press), p. 186

Wada, K., Papadopoulos, P., \& Spaans, M. 2009, ApJ, 702, 63 